\title{
Uso de modelos matemáticos para el cálculo de ruido en las principales avenidas de Panamá
}

\section{Use of mathematical models for the calculation of noise in the main streets of Panamá}

\begin{abstract}
Joan Quintero ${ }^{l}$; Edwin De Frias ${ }^{l}$; Félix Henríquez ${ }^{2 *}$
${ }^{1}$ Licenciatura en Ingeniería en Mecánica - Facultad de Ingeniería Mecánica-Universidad Tecnológica de Panamá,

${ }^{2}$ Departamento de Energía y Ambiente - Facultad de Ingeniería Mecánica-Universidad Tecnológica de Panamá

Resumen Este artículo presenta resultados del cálculo de los niveles de emisión sonora provocados por el tráfico rodante o vehicular en las avenidas principales de la ciudad de Panamá, mediante el uso de modelos logarítmicos matemáticos. Se utilizaron cuatro modelos matemáticos para calcular los niveles de emisión sonora y, posteriormente, se compararon los resultados con los resultados de los estudios realizados por la Facultad de Ingeniería Mecánica de la UTP "Muestreo con sonómetros (utilizando sonómetro) y Mapa de Ruido utilizando el modelo matemático SoundPLan versión 6.4". Los modelos logarítmicos matemáticos seleccionados fueron en modelos FHWA de Estados Unidos, el modelo de Valdivia de Chile, el NMPB Routes-96 de Francia y el modelo RLS90 de Alemania. Luego de comparar los resultados de los cuatro modelos logarítmicos matemáticos, con los resultados de los estudios realizados en UTP, se calculó el porcentaje de error de cada modelo logarítmico matemático. Con base en los resultados del porcentaje de error de modelos matemáticos, se validó el uso de tres de modelos matemáticos para el cálculo de ruido para Panamá, los modelos FHWA, Valdivia y RLS90. Por otro lado, para las condiciones de Panamá, no se recomienda el uso del modelo NMPB Routes-96.
\end{abstract}

Palabras Clave Nivel de emisión sonora, aforos vehiculares, FHWA, RLS 90, NMPB Routes-96, Valdivia.

\begin{abstract}
This article presents results of the calculation of sound emission levels caused by vehicular traffic in the main streets of Panama City, using mathematical logarithmic models. Four mathematical models were used to calculate the levels of sound emission levels and, later, the results were compared with the results of the studies carried out by the Faculty of Mechanical Engineering of the UTP "Sampling with sound level meters (using sound level meter) and Noise Map using the mathematical model SoundPLan version 6.4 ". The mathematical logarithmic models selected were in USA FHWA models, the Valdivia model in Chile, the NMPB Routes-96 in France and RLS90 in Germany. After comparing the results of the four mathematical logarithmic models, with the results of the studies carried out in UTP, the error percentage of each mathematical logarithmic model was calculated. Based on the results of the error percentage of mathematical models, the use of three mathematical models was validated for the calculation of noise for Panama, the FHWA, Valdivia and RLS90 models. While for Panama conditions, the use of the NMPB Routes-96 model is not recommended.
\end{abstract}

Keywords Sound emission level, vehicular gauges, FHWA, RLS 90, NMPB Routes-96, Valdivia.

*Corresponding Author:felix.henriquez@utp.ac.pa

\section{Introducción}

El aumento del tráfico vehicular en la Ciudad de Panamáha ocasionado un alza en los índices de los niveles de ruido, a tal punto que el tráfico rodado llega a constituir la principal fuente de contaminación acústica en las ciudades del país. La predicción del ruido de tráfico permite obtener datos que pueden considerarse en la planificación de las redes viales. Sin embargo, la gran cantidad de variables que intervienen en esta predicción requiere que los modelos predictivos sean probados y ajustados para las realidades locales con el fin de que los valores obtenidos sean confiables.
En este trabajo se analiza la aplicación de diferentes modelos predictivos de ruido de tráfico, originados en diversos estudios, donde se ha utilizado y aceptado estos modelos. Este análisis permite establecer el modelo predictivo de mejor desempeño a través de una validación con mediciones en vías estudiadas dentro de la misma ciudad, y de acuerdo a las características locales y su parque automotriz.

\subsection{Sonido y la contaminación acústica}

Sonido es una forma de energía producido por la vibración de un objeto que al hacerlo crea un movimiento de las moléculas de medios elásticos, como el aire. Oímos ondas 
cuando las moléculas perturbadas moviéndose por el aire, llegan a nuestros tímpanos, haciéndolos vibrar y enviando al cerebro debilísimos impulsos de energía electroquímica donde serán interpretados [1].

Según estudios realizados por la Organización Mundial de la Salud, el ruido ha sido catalogado como la primera molestia ambiental en los países industrializados. Es una característica común de la vida actual que, aunque molesta y dañina, puede llegar a considerarse inherente al desarrollo de cualquier tipo de actividad.

Podemos definir entonces la contaminación acústica como la presencia en el ambiente de ruidos o vibraciones, cualquiera que sea el emisor acústico que los origine, que impliquen molestia, riesgo o daño para las personas, para el desarrollode sus actividades o para los bienes de cualquier naturaleza, o que causen efectos significativos sobre el medio ambiente.

La exposición prolongada a altos niveles de ruido produce efectos negativos en la salud como la pérdida parcial o total de la audición, el estrés, problemas cardiacos e insomnio y el medio ambiente.

\section{Metodología}

La metodología utilizada en esta investigación estuvo compuesta por los siguientes puntos: selección de puntos para cálculo de ruido mediante modelos matemáticos, selección de los modelos matemáticos, análisis de resultados y cálculo de porcentaje de error de los modelos comparados con investigaciones previas y validación de los modelos matemáticos.

\subsection{Selección de puntos para cálculo de ruido mediante modelos matemáticos}

Al momento de seleccionar los puntos en las avenidas para calcular los niveles de ruido usando modelos matemáticas se tomaron en cuenta los siguientes factores:

- Análisis de los datos proporcionados por la Autoridad de Tránsito y Transporte Terrestre (ATTT).

- Los sitios donde se requiere un bajo nivel sonoro como lo son hospitales y colegios, localizados cerca de una vía con alto flujo vehicular.

- Localización de los sectores de desarrollo industrial.

- Puntos monitoreados en las tesis: "Monitoreo de la contaminación acústica en sitio crítico de la ciudad de Panamá" y "Mapa de Ruido utilizando el modelo matemático SoundPLan versión 6.4”, realizadas por estudiantes de la Universidad Tecnológica de Panamá en el año 2007.

- Limitaciones como la falta de datos actualizados de aforos en el área metropolitana por parte la Autoridad de la Tránsito y Transporte Terrestre (ATTT), que concuerden con los puntos monitoreados en 2007.
Además, se delimitó el estudio a un periodo de 12 horas dividido en grupos de horas picos o de alto flujo vehicular. Estos periodos de horas picos son siguientes:

- $\quad$ El periodo de 6:30 a. m. a 8:30 a. m., donde se desplaza la población hacia el sector laboral y el sector educativo.

- $\quad$ El periodo entre las 11:30 a. m. y 1:30 p. m. donde el desplazamiento se intensifica debido al cambio de turno en escuelas públicas, y la movilización hacia restaurantes, por las horas de almuerzo del sector laboral.

- El periodo entre las 3:45 p. m. y 5:45 p. m., donde se desplaza la población de vuelta a sus hogares, y hacia diversas actividades de recreación.

Tomando en cuenta estos factores se seleccionaron los siguientes 29 puntos para calcular los niveles de ruido:

1. Ave. Ricardo J. Alfaro y entrada a Residencial ElBosque.

2. Ave. Fernández de Córdoba y Calle 77 A Oeste.

3. Vía España y Calle 49.

4. Ave. Simón Bolívar y Calle 64 Oeste.

5. Ave. Perú y Calle 37 Este.

6. Vía España y Ave. Fernández de Córdoba.

7. Vía España, Ave. 12 de Octubre, Ave. Ernesto T. Lefevre y Calle 81 Este.

8. Ave. Vía España y 12 de Octubre.

9. Ave. Balboa y Ave. Federico Boyd.

10. Vía España, Ave. Ira B Norte y Calle 62.

11. Ave. Simón Bolívar, Ave. Ramón Arias y Calle El Paical.

12. Ave. Simón Bolívar y Calle 62 C Oeste.

13. Vía España y Belisario Porras.

14. Ave. Simón Bolívar y Ave. 12 C Norte.

15. Ave. Simón Bolívar y Fernández de Córdoba.

16. Ave. Simón Bolívar y Ave. 8va C Norte.

17. Ave. Simón Bolívar, Ave. 14 C Norte y Ave. 12 de Octubre.

18. Paso vehicular de San Miguelito.

19. Simón Bolívar y 12 de Octubre.

20. Ave. Fernández de Córdoba y 12 de Octubre.

21. Ave. Manuel E. Batista, Ave. José A. Fábrega y Eusebio A. Morales.

22. Ave. Ricardo J. Alfaro y Calle 74 Oeste.

23. Vía España, Ave. Brasil y Ave. Ramón Arias.

24. Ave. Ricardo J. Alfaro, Ave. Makario III de Chipre y Calle 62 A Oeste.

25. Ave. Ricardo J. Alfaro, Ave. 23 Norte y Ave. Ira Norte.

26. Ave. Fernández de Córdoba y Calle 64 Oeste.

27. Ave. Fernández de Córdoba y Calle 66 Lucas Zarak.

28. Ave. Ricardo J. Alfaro y entrada a Linda Vista.

29. Ave. Cincuentenario y Ave. Domingo Díaz (Sobre la Ave. José Agustín Arango) a 500 metros del puente sobre el río Juan Díaz. 


\subsection{Selección de los modelos matemáticos}

Los modelos matemáticos se dividen en simples y complejos y su selección depende de la utilidad y veracidad de los resultados a obtener. Estos modelos de previsión, que están conformados por ecuaciones matemáticas sencillas, se elaboran a partir de la integración de dos modelos, el modelo de fuente y el modelo de propagación. Los modelos de fuente son esencialmente empíricos y conllevan una concepción estadística del fenómeno, puesto que el conocimiento de la potencia acústica y la directividad de cada vehículo son una tarea inabordable e injustificada [2].

Se seleccionaron cuatro modelos para estudiar en esta investigación: el Modelo FHWA de USA, el Modelo de Valdivia de Chile, el Modelo NMPB-Routes-96 de Francia y el Modelo RLS90 de Alemania.

A continuación, se especifican los modelos de ruido de tráfico utilizados en esta investigación.

\section{Modelo FHWA}

Ecuación del modelo FHWA para el cálculo del nivel de emisión sonora de referencia a 15.2 metros $\left(\mathrm{dB}_{\mathrm{A}}\right)$. $\left.\begin{array}{l}\mathrm{LRE}=10 \log \left(10^{(0.1 * \operatorname{Leq}(\mathrm{d}) \text { auto })}+10^{(0.1 * \text { Leq (d) mediano })}+10^{(0.1 * \operatorname{Leq}(\mathrm{d})}\right. \\ \text { pesado })\end{array}\right)$

Donde:

$\operatorname{Leq}(\mathrm{d}) \mathrm{i}=\left(\mathrm{L}_{0}\right) \mathrm{Ei}+(\Delta$ tráfico $) \mathrm{i}+(\Delta$ distancia $) \mathrm{i}+$

$(\Delta$ pendiente $) \mathrm{i}+(\Delta$ segmento $) \mathrm{i}$

$\left(\mathrm{L}_{0}\right)$ Ei: nivel equivalente de emisión $\left(\mathrm{dB}_{\mathrm{A}}\right)$ para el tipo i de vehículo (vi): correcciones del tipo i de vehículo [3].

\section{Correcciones del tipo i de vehículo}

Automóviles:

(L ) $\mathrm{E}=38.1 * \log (\mathrm{v})-2.4 \mathrm{~dB}(\mathrm{~A})$

Vehículos medianos:

(L ) $\mathrm{E}=33.9 * \log (\mathrm{v})+16.4 \mathrm{~dB}(\underset{\mathrm{A}}{\mathrm{f}})$

Camiones pesados:

$$
\left(\mathrm{L}_{0}\right) \mathrm{E}=24.6 * \log (\mathrm{v})+38.5 \mathrm{~dB}\left(\mathrm{~A}_{\mathrm{A}}\right)
$$

Donde: v es la velocidad media de los vehículos [Km/

\section{h] Corrección por intensidad de tráfico}

( $\Delta$ tráfico $) \mathrm{i}=10 \log ((\mathrm{Ni} *$ do $) / \mathrm{vi})-25 \mathrm{~dB}\left({ }_{\mathrm{A}}\right)$

- do: distancia de referencia 15.2 [m].

- Ni: intensidad horaria de vehículos tipo i [veh/h].

- $\quad$ vi: velocidad del vehículo tipo i.

\section{Corrección por distancia a la fuente y tipo de suelo}

$(\Delta$ distancia $)=10 \log (\mathrm{do} / \mathrm{d})^{1+\alpha}$
Donde d es la distancia perpendicular entre la línea central del carril al observador y $\alpha$ es parámetro de sitio cuyo valor depende en las condiciones del sitio.

$(\Delta$ tráfico $) \mathrm{i}=10 \log ((\mathrm{Ni} * \mathrm{do}) / \mathrm{vi})-25 \mathrm{~dB}(\mathrm{~A})$

\section{Corrección por rampa, pendiente de carretera}

Tabla 1. Corrección por rampa

\begin{tabular}{|l|l|}
\hline 0 a $2 \%$ Dgr & $0 \mathrm{~dB}(\mathrm{~A})$ \\
\hline 3 a $4 \%$ Dgr & $+2 \mathrm{~dB}\left({ }_{\mathrm{A}}\right)$ \\
\hline 5 a $6 \%$ Dgr & $+3 \mathrm{~dB}\left({ }_{\mathrm{A}}\right)$ \\
\hline Más de $7 \%$ Dgr & $+5 \mathrm{~dB}\left({ }_{\mathrm{A}}\right)$ \\
\hline
\end{tabular}

\section{Modelo de la ciudad de Valdivia}

Ecuación del modelo de Valdivia Chile para el cálculo del nivel de emisión sonora de referencia a 25 metros $\left(\mathrm{dB}_{\mathrm{A}}\right)$ [4].

$$
\mathrm{LRE}=33.6+10 \cdot \log \left(\mathrm{Q}_{l}+9.2 \cdot \mathrm{Q}_{p}+6 \cdot \mathrm{Q}_{b}\right)+C_{v e l}+C_{p a v}
$$

Donde:

LRE: Nivel de emisión de referencia a 25 metros $\left(\mathrm{dB}_{\mathrm{A}}\right)$. $\mathrm{Q}_{l}, \mathrm{Q}_{p} \mathrm{y} Q_{b}$ : Flujos de vehículos livianos, pesados y buses respectivamente.

$C_{\text {vel }}$ : Corrección por velocidad de circulación $\left(\mathrm{dB}_{\mathrm{A}}\right)$.

$C_{\text {pav }}$ : Corrección por tipo de carpeta de rodado $\left(\mathrm{dB}_{\mathrm{A}}\right)$.

Tabla 2. Valores $C_{\text {vel }}$ para distintas velocidades de circulación

\begin{tabular}{|l|l|l|l|l|l|}
\hline $\begin{array}{l}\text { Velocidad } \\
(\mathrm{km} / \mathrm{h})\end{array}$ & $<50$ & 60 & 70 & 80 & 90 \\
\hline$C_{\text {vel }}\left(d B_{A}\right)$ & 0 & 1 & 2 & 3 & 4 \\
\hline
\end{tabular}

Tabla 3. Valores $C_{\text {pav }}$ según el tipo de calzada

\begin{tabular}{|l|l|l|l|l|}
\hline $\begin{array}{l}\text { Tipo de } \\
\text { pavimento }\end{array}$ & $\begin{array}{l}\text { Asfalto } \\
\text { liso }\end{array}$ & $\begin{array}{l}\text { Asfalto } \\
\text { rugoso }\end{array}$ & Hormigón & Adoquinado \\
\hline$C_{\text {pav }}\left(d B_{A}\right)$ & -0.5 & 0 & 1.5 & 4 \\
\hline
\end{tabular}

\section{Modelo NMPB-ROUTES-96}

En la actualidad el método de evaluación recomendado según la legislación europea para países miembros sin métodos nacionales o para los que quieren cambiarlo. [5]

$L_{c} e q=18+10 \log \left(Q_{L}+E Q_{P}\right)+20 \log V-12 \log (d+$
$-30 \log$

Donde:

$L_{e q}=$ Nivel Sonoro Continuo equivalente $\left(\mathrm{dB}_{\mathrm{A}}\right)$ 
$Q_{L}=$ Caudal Vehículos Ligeros $(\mathrm{veh} / \mathrm{h})($ carga total $\leq 3500$ $\mathrm{kg})$

$Q_{P}=$ Caudal Vehículos Pesados (veh/h) (carga total > $3500 \mathrm{~kg}$ )

$\mathrm{V}=$ Velocidad media de los vehículos $(\mathrm{km} / \mathrm{h})$

$\mathrm{Lc}=$ Anchura de la calzada $(\mathrm{m})$

$\mathrm{E}=$ Factor de Equivalencia Acústica entre vehículos ligeros y pesados, es función del porcentaje de rampa y del tipo de vía.

Tabla 4. Factor de equivalencia acústica

\begin{tabular}{|c||c||c||c||c|}
\hline \% Pendiente & $\leqslant \mathbf{2 \%}$ & $\mathbf{3 \%}$ & $\mathbf{4 \%}$ & $\mathbf{5 \%}$ \\
\hline \hline Autovía o autopista & $\mathrm{E}=4$ & 5 & 5 & 6 \\
\hline \hline Vía rápida o urbana & 7 & 9 & 10 & 11 \\
\hline \hline Vía urbana & 10 & 13 & 16 & 18 \\
\hline
\end{tabular}

\section{Modelo RLS90}

Ecuación del modelo RLS 90 para el cálculo del nivel de emisión sonora de referencia a 25 metros $\left(\mathrm{dB}_{\mathrm{A}}\right)$. [6]

Leq $(\mathrm{A})=10^{*} \log \left[10^{\wedge}(\mathrm{LMi} / 10)\right]$

Donde:

Leq: suma energética de los niveles puntuales LMi

LMi: nivel aportado por cada fuente puntual i

$\mathrm{LME}=\mathrm{L} 25+C_{\text {vel }}+C_{\text {caaaeta }}+C_{\text {gaaaiente }}$

$\mathrm{L} 25=37.3+10 * \log (\mathrm{M} *(1+0.082 * \mathrm{P}))$

Donde:

L25: Nivel Sonoro Base a 25 metros. $\left(\mathrm{dB}_{\mathrm{A}}\right)$

M: Densidad de tráfico. (Vehículos /hora)

P: Porcentaje de vehículos pesados. (\%)

\section{Corrección por velocidad}

$$
\begin{aligned}
& C_{\text {vel }}=L_{\text {Ligeros }}-37.3+10 *\left(\log \left[\frac{100+\left(10^{0.1 * C}\right) * P}{100+8.23 * P}\right]\right. \\
& L_{\text {Ligeros }}=27.8+10 * \log \left(1+\left(0.02 * V_{\text {Ligeros }}\right)^{3}\right) \\
& L_{\text {Pesados }}=23.1+12.5 * \log \left(V_{\text {Pesados }}\right) \\
& C \stackrel{=}{=} L_{\text {Pesados }}-L_{\text {Ligeros }}
\end{aligned}
$$

Donde:

$C_{\text {vel }}$ : Corrección por velocidad de los vehículos (dB)

P: Porcentaje de vehículos pesados. (\%)

$\mathrm{V}_{\text {Ligeros }}$ : Velocidad vehículos livianos. (Vehículos/hora) (60 $\mathrm{Km} / \mathrm{h}$ )

$\mathrm{V}_{\text {Pesados: }}$ Velocidad vehículos pesados. (Vehículos /hora) (40 $\mathrm{Km} / \mathrm{h}$ )

\section{Corrección por tipo de carpeta}

Tabla 5. Corrección por tipo de carpeta Modelo RLS-90

\begin{tabular}{|l|c|c|c|c|}
\hline \multicolumn{1}{|c|}{ Tipo de superficie } & \multicolumn{4}{c|}{$\begin{array}{c}\text { Máxima velocidad } \\
\text { permitida en Km/hora }\end{array}$} \\
\hline & $\mathbf{3 0}$ & $\mathbf{4 0}$ & $\mathbf{7 5 0}$ & $\mathbf{7 0 0}$ \\
\hline Asfalto no ranurado & 0 & 0 & 0 & 0 \\
\hline Concreto o asfalto ranurado & 1 & 1,5 & 2 & 2 \\
\hline Superficie pedregosa con textura lisa & 2 & 2,5 & 3 & 3 \\
\hline Superficie pedregosa con estructura rugosa & 3 & 4,5 & 6 & 6 \\
\hline Hormigón con tratamiento escoba metal & 1 & 1 & 1 & 1 \\
\hline Asfalto hormigón sin grietas & 0 & 0 & 0 & -2 \\
\hline Asfalto poroso con más de 15\% de poros del tipo 0/11 & 0 & 0 & 0 & -4 \\
\hline Asfalto poroso con más de 15\% de poros del tipo 0/8 & 0 & 0 & 0 & -5 \\
\hline
\end{tabular}

\section{Corrección por gradiente}

$C_{\text {gradiente }}=0.6 *|G|-3$ para gradientes $>5 \%$

Para gradientes menores a 5\% la corrección de gradiente es 0 $\mathrm{dB}_{\mathrm{A}}$.

\subsection{Análisis de resultados y cálculo de porcentaje de error de los modelos comparados con investigaciones previas}

Los resultados de las 29 avenidas fueron comparados con las normas y decretos nacionales e internacionales con el fin de verificar el cumplimiento de las mismas. A continuación, en el punto 3 se muestran los gráficos de los resultados a detalle. Además, se seleccionaron cinco de las veintinueve avenidas para compararlas con los resultados de los estudios realizados en la Universidad Tecnológica de Panamá: "Monitoreo de la contaminación acústica en sitio crítico de la ciudad de Panamá" y "Mapa de ruido utilizando el modelo matemático SoundPLan versión 6.4".

\subsection{Validación de los modelos matemáticos}

Para validar el uso de los modelos matemáticos se procedió a calcular el porcentaje de error de modelo comparado con los resultados de los estudios previos realizados en la Universidad Tecnológica de Panamá. Una vez calculado los porcentajes de error se pudo validar el uso de tres modelos matemáticos, el Modelo FHWA de USA, el Modelo de Valdivia de Chile y el Modelo RLS-90 de Alemania para el cálculo de ruido para Panamá. No se recomienda el uso del Modelo NMPB-Routes-96 para Panamá, ya que dicho modelo presenta el más alto porcentaje de error debido a la gran cantidad de correcciones.

A continuación, se presenta el cálculo de porcentaje de error de los modelos matemáticos versus los resultados de los estudios: "Monitoreo de la contaminación acústica en sitios críticos de la ciudad de Panamá" y "Mapa de ruido utilizando el modelo matemático SoundPlan versión 6.4". 
Quintero (et al): Uso de modelos matemáticos para el cálculo de ruido en las principales avenidas de Panamá

Tabla 6. Cálculo del portaje de error de los resultados de los modelos matemáticos

\begin{tabular}{|c|c|c|c|c|c|}
\hline Estudios & $\begin{array}{c}\text { \%Error } \\
\text { RLS-90 }\end{array}$ & $\begin{array}{c}\text { \%Error } \\
\text { NMPB- } \\
\text { Routes-96 }\end{array}$ & $\begin{array}{c}\text { \%Error } \\
\text { FHWA }\end{array}$ & $\begin{array}{c}\text { \%Error } \\
\text { Valdivia }\end{array}$ \\
\hline Monitoreo & 2.27 & 3.75 & 4.57 & & 2.08 \\
\hline SoundPlan & 4.27 & 7.68 & 1.06 & & 3.71 \\
\hline
\end{tabular}

\section{Resultados}

En las figuras 1 a 4 se puede observar de manera resumida el comportamiento de los diferentes modelos matemáticos comparados con las normas y decretos nacionales e internacionales. La Organización Mundial de la Salud recomienda que los niveles de exposición al ruido no deben sobrepasar los $70 \mathrm{~dB}_{\mathrm{A}}$. Por otro lado, el Ministerio de Salud (MINSA) en el Decreto Ejecutivo $N^{\circ} 1$ del 15 de enero del 2004 determina que los niveles de ruido para áreas residenciales e industrias de 6:00 a. m. a 9:59 p. m. no deben exceder los $60 \mathrm{~dB}_{\mathrm{A}}$, y El Reglamento COPANIT 44-2000 recomienda un nivel máximo de ruido de $85 \mathrm{~dB}_{\mathrm{A}}$ para una jornada de trabajo de 8 horas.

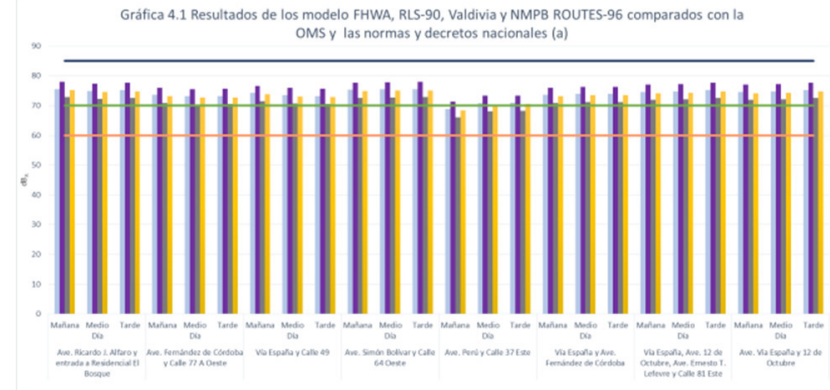

Figura 1. Resultados del modelo FHWA, RLS-90, Valdivia y NMPB ROUTES-96 comparados con la OMS y las normas ydecretos nacionales (a). Gráfica 4.2 Resultados de los modelo FHWA, RLS-90, Valdivia y NMPB ROUTES-96 comparados con la
OMSy las normas y decretos nacionales (b)

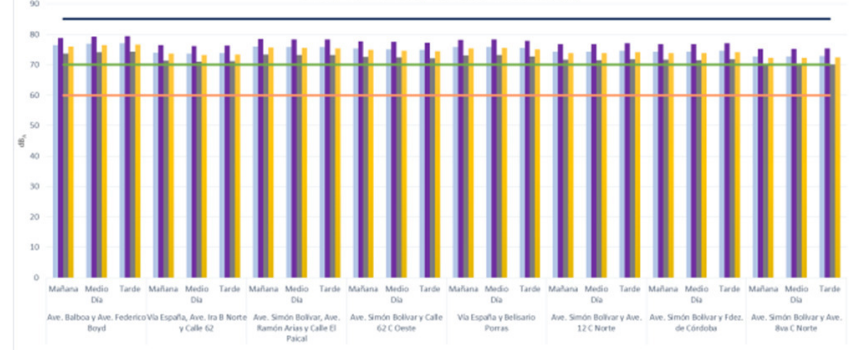

Figura 2. Resultados del modelo FHWA, RLS-90, Valdivia y NMPB ROUTES-96 comparados con la OMS y las normas y decretos nacionales(b).

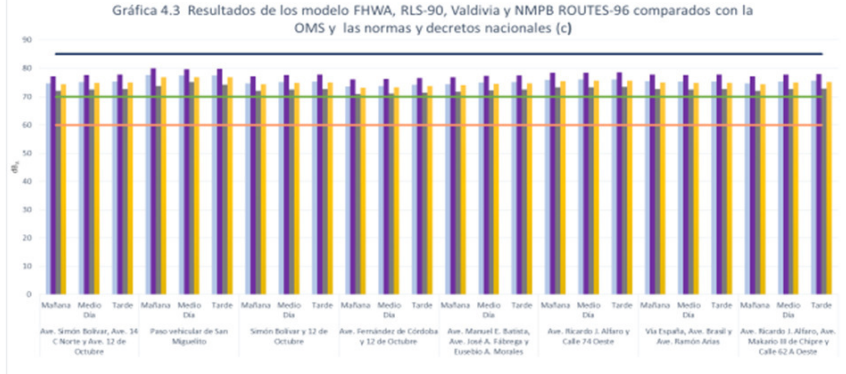

Figura 3. Resultados de los modelos FHWA, RLS-90, Valdivia y NMPB ROUTES-96 comparados con la OMS y las normas ydecretos nacionales (c).

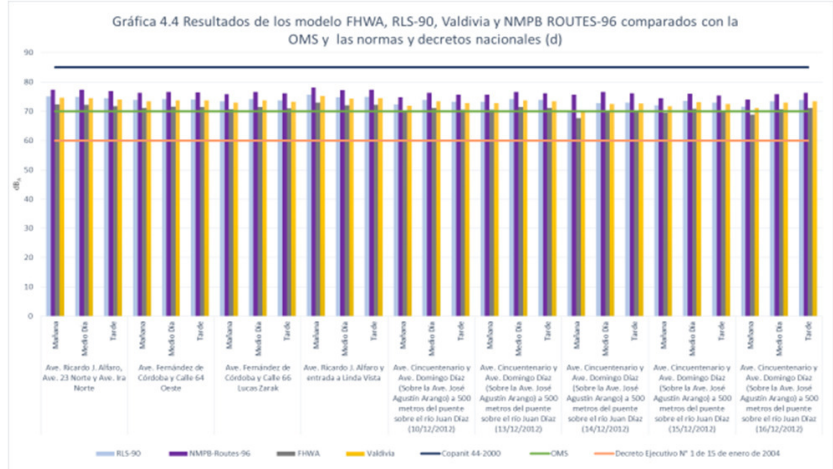

Figura 4. Resultados del modelo FHWA, RLS-90, Valdivia y NMPB ROUTES-96 comparados con la OMS y las normas y decretos nacionales(d).

En la tabla 7 se muestran los resultados de los modelos matemáticos comparados con los resultados del estudio "Monitoreo de la contaminación acústica en sitio crítico de la Ciudad de Panamá" realizado en la Universidad Tecnológica de Panamá, en el 2007 por Escobar M., Salomón RodríguezS.

Tabla 7. Resultados de modelos matemáticos comparados con monitoreo de la contaminación acústica en sitios críticos de la Ciudad de Panamá

\begin{tabular}{|c|c|c|c|c|c|c|}
\hline \multirow{2}{*}{ Intersección } & \multirow{2}{*}{ Hora } & RLS90 & $\begin{array}{l}\text { NMPB- } \\
\text { Routes- } \\
96 \\
\end{array}$ & FHWA & aldivia & Monitoreo \\
\hline & & $\begin{array}{c}\operatorname{Leq}(A) \\
d_{B}\end{array}$ & $\begin{array}{c}\operatorname{Leq}(A) \\
d B_{A}\end{array}$ & $\begin{array}{c}\operatorname{Leq}(A) \\
d_{B}\end{array}$ & $\begin{array}{c}\operatorname{Leq}(A) \\
d_{B}\end{array}$ & $\begin{array}{c}\operatorname{Leq}(A) \\
d_{B}\end{array}$ \\
\hline \multirow{3}{*}{$\begin{array}{c}\text { Ave. Balboa } \\
\text { y Ave. } \\
\text { Federico } \\
\text { Boyd }\end{array}$} & Mañana & 76.40 & 78.82 & 72.04 & 75.97 & 73.2 \\
\hline & $\begin{array}{c}\text { Medio } \\
\text { Día }\end{array}$ & 76.85 & 79.27 & 74.25 & 76.42 & 73.85 \\
\hline & Tarde & 77.00 & 79.42 & 74.40 & 76.57 & 72.3 \\
\hline \multirow{3}{*}{$\begin{array}{l}\text { Vía España } \\
\text { y } 12 \text { de } \\
\text { Octubre }\end{array}$} & Mañana & 74.60 & 77.02 & 70.24 & 74.17 & 75.65 \\
\hline & $\begin{array}{c}\text { Medio } \\
\text { Día }\end{array}$ & 74.73 & 77.16 & 72.13 & 74.3 & 76.05 \\
\hline & Tarde & 77.36 & 77.62 & 72.60 & 74.77 & 75.2 \\
\hline \multirow{2}{*}{$\begin{array}{l}\text { Vía Simón } \\
\text { Bolívar y } \\
\text { Fdez. de } \\
\text { Córdoba }\end{array}$} & Mañana & 74.30 & 76.72 & 69.94 & 73.87 & 75 \\
\hline & $\begin{array}{c}\text { Medio } \\
\text { Día }\end{array}$ & 74.27 & 76.69 & 71.67 & 73.84 & 74.1 \\
\hline
\end{tabular}


Quintero (et al): Uso de modelos matemáticos para el cálculo de ruido en las principales avenidas de Panamá

\begin{tabular}{|c|c|c|c|c|c|c|}
\hline & Tarde & 74.58 & 77.01 & 71.98 & 74.15 & 74.75 \\
\hline \multirow{4}{*}{$\begin{array}{c}\text { Paso } \\
\text { vehicular de } \\
\text { San } \\
\text { Miguelito }\end{array}$} & Mañana & 77.59 & 79.98 & 72.49 & 76.94 & 78.4 \\
\cline { 2 - 7 } & Medio & 77.43 & 79.75 & 75.21 & 76.8 & 76.67 \\
\hline & Tarde & 77.50 & 79.87 & 74.36 & 76.83 & 76.35 \\
\hline \multirow{2}{*}{$\begin{array}{c}\text { Vía Simón } \\
\text { Bolívar y 12 } \\
\text { de Octubre }\end{array}$} & \begin{tabular}{c} 
Mañana \\
\cline { 2 - 7 }
\end{tabular} & 74.75 & 77.18 & 70.39 & 74.32 & 74.5 \\
\cline { 2 - 7 } & Tarde & 75.20 & 77.63 & 72.60 & 74.77 & 78.5 \\
\hline
\end{tabular}

A continuación, se muestran los resultados de modelos matemáticos comparados con los resultados del estudio "Mapa de ruido utilizando el modelo matemático SoundPlan versión 6.4", realizado en la Universidad Tecnológica de Panamá, en 2009 por Matthews D., Obando E.

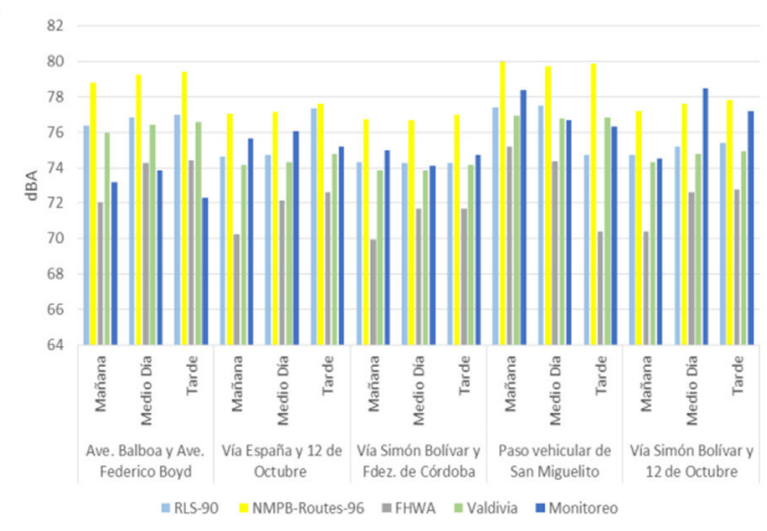

Figura 5. Resultados de modelos matemático vs. Monitoreo de la contaminación acústica en sitios críticos de la ciudad de Panamá.

Table 8. Resultados de Modelo FHWA comparado con Mapa de ruido utilizando el modelo matemático SoundPlan versión 6.4

\begin{tabular}{|l|c|c|c|c|}
\hline \multicolumn{1}{|c|}{ INTERSECCIÓN } & \multicolumn{3}{|c|}{ Modelo RLS90 } & $\begin{array}{c}\text { SOUNDP } \\
\text { LAN }\end{array}$ \\
\hline & $\begin{array}{c}\text { Mañana } \\
\mathrm{dB}_{\mathrm{A}}\end{array}$ & $\begin{array}{c}\text { Medio } \\
\text { día } \\
\mathrm{dB}_{\mathrm{A}}\end{array}$ & $\begin{array}{c}\text { Tarde } \\
\mathrm{dB}_{\mathrm{A}}\end{array}$ & $\begin{array}{c}\mathrm{Lm}^{25} \\
\text { (día) } \mathrm{dB}_{\mathrm{A}}\end{array}$ \\
\hline $\begin{array}{l}\text { Ave. Balboa y Ave. Federico } \\
\text { Boyd }\end{array}$ & 76.40 & 76.85 & 77.00 & 74 \\
\hline Vía España y 12 de Octubre & 74.60 & 74.73 & 77.36 & 70.7 \\
\hline $\begin{array}{l}\text { Vía Simón Bolívar y Fdez. de } \\
\text { Córdoba }\end{array}$ & 74.30 & 74.27 & 74.58 & 72.7 \\
\hline Paso vehicular de San Miguelito & 77.59 & 77.43 & 77.50 & 73.4 \\
& & & & \\
\hline
\end{tabular}

Tabla 9. Resultados de Modelo Valdivia comparado con Mapa de ruido utilizando el modelo matemático SoundPlan versión 6.4

\begin{tabular}{|c|c|c|c|c|}
\hline INTERSECCIÓN & \multicolumn{3}{|c|}{ Modelo FHWA } & $\begin{array}{c}\text { SOUND } \\
\text { PLAN }\end{array}$ \\
\hline & $\begin{array}{c}\text { Mañan } \\
\text { a } \\
\mathrm{dB}_{\mathrm{A}}\end{array}$ & $\begin{array}{c}\text { Medio } \\
\text { día } \\
\mathrm{dB}_{\mathrm{A}}\end{array}$ & $\begin{array}{c}\text { Tarde } \\
\mathrm{dB}_{\mathrm{A}}\end{array}$ & $\begin{array}{c}\text { Lm25 } \\
\text { (día) } \\
\mathrm{dB}_{\mathrm{A}}\end{array}$ \\
\hline $\begin{array}{c}\text { Ave. Balboa y Ave. Federico } \\
\text { Boyd }\end{array}$ & 72.04 & 74.25 & 74.4 & 74 \\
\hline $\begin{array}{c}\text { Ave. Vía España y 12 de } \\
\text { Octubre }\end{array}$ & 70.24 & 72.13 & 72.6 & 70.7 \\
\hline $\begin{array}{c}\text { Ave. Simón Bolívar y Fdez. } \\
\text { de Córdoba }\end{array}$ & 69.94 & 71.67 & 71.98 & 72.7 \\
\hline $\begin{array}{c}\text { Paso vehicular de San } \\
\text { Miguelito }\end{array}$ & 72.49 & 75.21 & 74.36 & 73.4 \\
\hline
\end{tabular}

Table 10. Resultados Modelo NMPB-Routes-96 comparado con Mapa de ruido utilizando el modelo matemático SoundPlan versión 6.4

\begin{tabular}{|c|c|c|c|c|}
\hline INTERSECCIÓN & \multicolumn{3}{|c|}{ Modelo Valdivia } & $\begin{array}{c}\text { SOUND } \\
\text { PLAN }\end{array}$ \\
\hline & $\begin{array}{c}\text { Mañan } \\
\text { a } \\
\mathrm{dB}_{\mathrm{A}}\end{array}$ & $\begin{array}{c}\text { Medio } \\
\text { día } \\
\mathrm{dB}_{\mathrm{A}}\end{array}$ & $\begin{array}{c}\text { Tarde } \\
\mathrm{dB}_{\mathrm{A}}\end{array}$ & $\begin{array}{c}\text { Lm25 } \\
\text { (día) } \\
\mathrm{dB}_{\mathrm{A}}\end{array}$ \\
\hline $\begin{array}{c}\text { Ave. Balboa y Ave. Federico } \\
\text { Boyd }\end{array}$ & 87.83 & 88.28 & 88.43 & 74 \\
\hline $\begin{array}{c}\text { Ave. Vía España y 12 de } \\
\text { Octubre }\end{array}$ & 86.03 & 86.16 & 86.63 & 70.7 \\
\hline $\begin{array}{c}\text { Ave. Simón Bolívar y Fdez. } \\
\text { de Córdoba }\end{array}$ & 85.73 & 85.7 & 86.01 & 72.7 \\
\hline $\begin{array}{c}\text { Paso vehicular de San } \\
\text { Miguelito }\end{array}$ & 88.99 & 88.76 & 88.87 & \\
\hline
\end{tabular}

Table 11. Resultados Modelo RLS-90 comparado con Mapa de ruido utilizando el modelo matemático SoundPlan versión 6.4

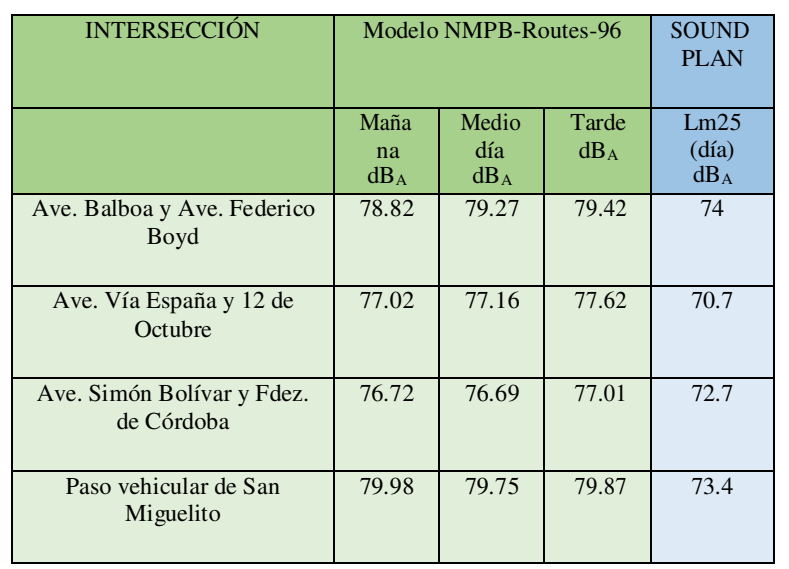

A continuación, se representan graficamente los resultados de los modelos matemáticos comparado con Mapa de Ruido utilizando el modelo matemático SoundPlan versión 6.4. 


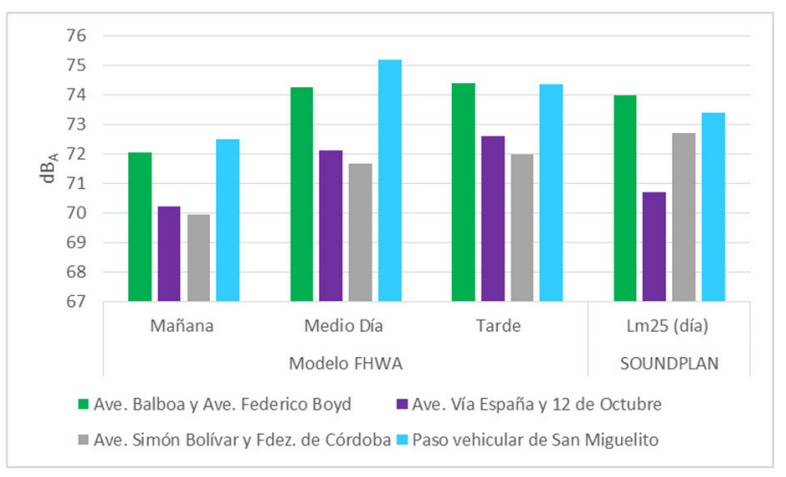

Figura 5. Resultados de Modelo FHWA vs. SOUNDPLAN.

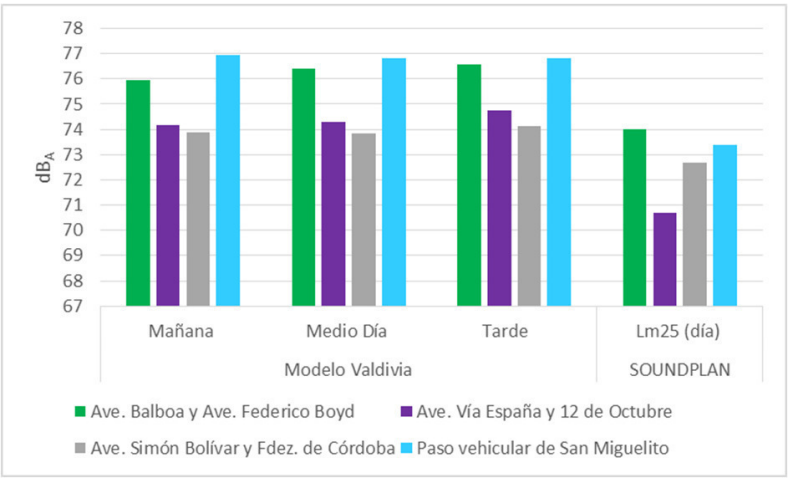

Figura 6. Resultados de Modelo Valdivia vs. SOUNDPLAN.

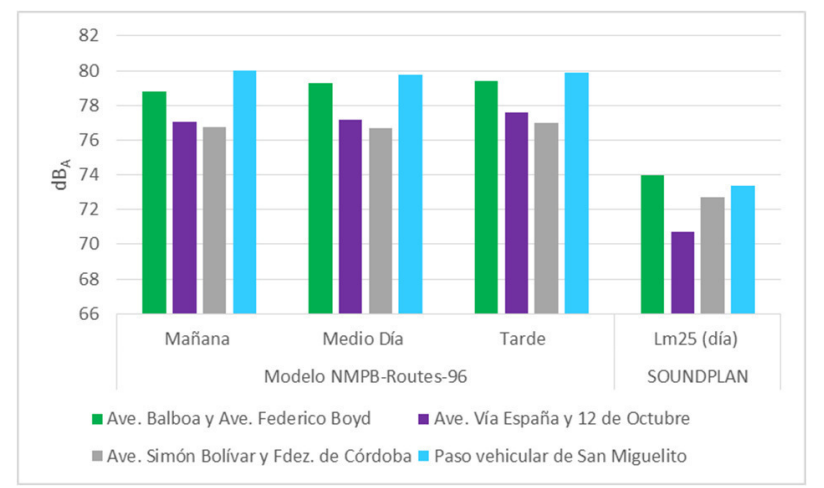

Figura 7. Resultados de Modelo NMPB-Routes-96 vs. SOUNDPLAN.

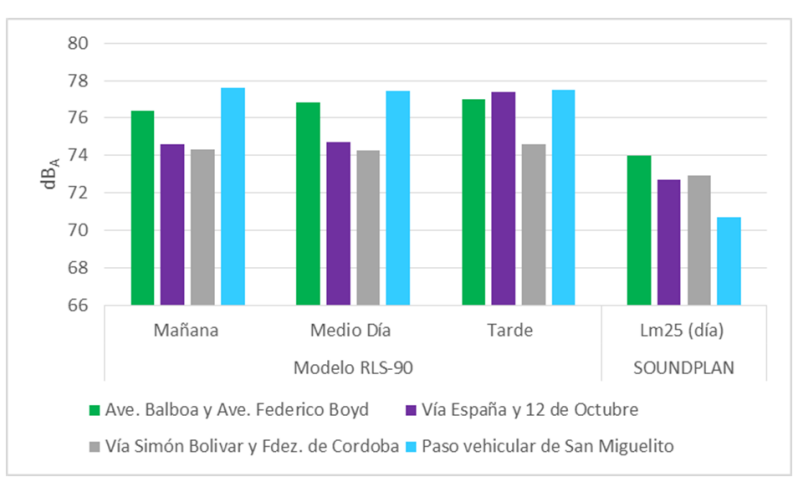

Figura 8. Resultados de Modelo RLS-90 vs. SOUNDPLAN.

\section{Conclusiones}

La emisión de ruido la ciudad de Panamá sobrepasa los niveles recomendados por la Organización Mundial de la Salud (70 dBA), y los niveles recomendados por Ministerio de Salud (MINSA) en el Decreto Ejecutivo $\mathrm{N}^{\circ} 1$ del 15 de enero del 2004 para una jornada de trabajo de 8 horas $(60 \mathrm{dBA})$.

El sitio con mayor contaminación acústica de acuerdo con esta investigación es el Paso vehicular de San Miguelito, dicho sitio contiene el mayor flujo de vehículos diarios.

No se cuenta con legislaciones eficientes para control y medición de la contaminación acústica por tráfico rodante en Panamá.

Este estudio permitió determinar que, no se considera la contaminación por ruido como un problema importante para los gobiernos e instituciones. Además, se desconocen los efectos a largo plazo la exposición a altos nivel de ruido porla población en general.

Se validó el uso de tres de modelos matemáticos para el cálculo de ruido para Panamá, los modelos FHWA de USA, Valdivia de Chile y RLS90 de Alemania.

Los resultados del modelo NMPB-Routes-96 presentan el mayor porcentaje de error comparado con ambos estudios, esto se debe a que hay realizar múltiples correcciones, por ende, no se recomienda su uso para las condiciones de Panamá.

\section{Recomendaciones}

Los modelos de predicción trabajan de la misma maneraque la medición. Se recomienda no superar distancias entre predicciones de 250 metros; ya que se puede alterar el resultado, debido a que puede variar el flujo vehicular y los porcentajes de ligeros y pesados.

Al hacer un estudio de contaminación acústica, esta información debe darse a conocer a las entidades gubernamentales encargadas del caso, para que ellas tomen las medidas necesarias sobre este problema.

Se deben aplicar medidas de prevención y reducción de emisiones de ruido por instituciones gubernamentales en la ciudad de Panamá.

Adoptar medidas para disminuir la emisión de ruido como la colocación de pantallas o barreras absorbentes de ondas acústicas, nuevas superficies de rodamiento y plantación de vegetación para absorción del sonido.

Reestructurar vías y planificar el desarrollo territorial para disminuir la alta concentración de tráfico vehicular y su consecuente emisión de ruido.

Para mejorar la eficiencia del algoritmo de predicción, sería importante crear un método para la ciudad de Panamá; ya que las características de todas las ciudades son diferentes y las correcciones se pueden modificar de acuerdo con la vía.

Los resultados de modelo de Valdivia de Chile, FHWA de USA y el modelo RLS-90 de Alemania presentan los porcentajes de exactitud más altos comparados con los 
resultados de monitoreo y SOUNDPLAN, por lo cual se recomienda su uso para el cálculo de ruido en Panamá.

\section{REFERENCIAS}

[1] Brennan, R. (1994). Diccionario Básico para la Actualidad Científica. Ponferrada, España: Editorial Celeste.

[2] Álvarez Rodenbeek, J. P., \& Suárez Silva, E. (2011). Estudio comparativo para modelos predictivos del ruido de tráfico rodado, a través de mediciones in situ en un sector de la ciudad de Osorno. Revistas Electrónicas UACH, 35.

[3] Alberto Ventoso del Rincón, David Martín Ruiz. (2016). MODELOS DE PREVISIÓN DE RUIDO. Pág. 7.

[4] Juan Pablo Álvarez Rodenbeek. (2010). Estudio comparativo para modelos predictivos del ruido de tráfico rodado, a través de mediciones in situ en un sector de la ciudad de Osorno. (Tesis de grado). Universidad Austral de Chile. Valdivia, Chile.

[5] Alberto Ventoso del Rincón, David Martín Ruiz. (2016). MODELOS DE PREVISIÓN DE RUIDO.

[6] Juan Pablo Álvarez Rodenbeek. (2010). Estudio comparativo para modelos predictivos del ruido de tráfico rodado, a través de mediciones in situ en un sector de la ciudad de Osorno. (Tesis de grado). Universidad Austral de Chile. Valdivia, Chile. 